\title{
Pre-reproductive stress in adolescent female rats alters oocyte microRNA expression and offspring phenotypes: pharmacological interventions and putative mechanisms
}

\author{
Hiba Zaidan', Dalia Galiani ${ }^{2}$ and Inna Gaisler-Salomon (1)
}

\begin{abstract}
Pre-reproductive stress (PRS) to adolescent female rats alters anxiogenic behavior in first (F1)- and second-generation (F2) offspring and increases mRNA expression of corticotropin-releasing factor receptor type 1 (Crhr1) in oocytes and in neonate offspring brain. Here, we ask whether the expression of Crhr1 and Crhr1-targeting microRNA is altered in brain, blood, and oocytes of exposed females and in the brain of their neonate and adult F1 and F2 offspring. In addition, we inquire whether maternal post-stress drug treatment reverses PRS-induced abnormalities in offspring. We find that PRS induces a selective increase in Crhr1-targeting mir-34a and mir-34c in blood and oocytes, while non-Crhr1 microRNA molecules remain unaltered. PRS induces similar microRNA changes in prefrontal cortex of F1 and F2 neonates. In adult animals, cortical Crhr1, but not mir-34, expression is affected by both maternal and direct stress exposure. PostPRS fluoxetine (FLX) treatment increases pup mortality, and both FLX and the Crhr1 antagonist NBI 27914 reverse some of the effects of PRS and also have independent effects on F1 behavior and gene expression. PRS also alters behavior as well as gene and miRNA expression patterns in paternally derived F2 offspring, producing effects that are different from those previously found in maternally derived F2 offspring. These findings extend current knowledge on interand trans-generational transfer of stress effects, point to microRNA changes in stress-exposed oocytes as a potential mechanism, and highlight the consequences of post-stress pharmacological interventions in adolescence.
\end{abstract}

\section{Introduction}

Exposure to an unpredictable, adverse environment has long-term effects on health, behavior, and endocrine function. Human and animal studies show that the effects of stress during or prior to gestation can propagate onto future generations ${ }^{1-4}$, and impact behavior and hypothalamic-pituitary-adrenal (HPA) axis function in offspring $^{5}$. The influences of early life stress on offspring behavior and neuroendocrine function have been reported for up to four generations ${ }^{6-11}$. The trans-generational

\footnotetext{
Correspondence: Inna Gaisler-Salomon (igsalomon@psy.haifa.ac.il)

${ }^{1}$ School of Psychological Sciences and the Integrated Brain and Behavior Research Center, University of Haifa, Haifa, Israel

${ }^{2}$ Department of Biological Regulation, Weizmann Institute of Science, Rehovot, Israel
}

effects of stress during adolescence, a period of profound changes to brain structure and function ${ }^{12,13}$, have been less extensively studied.

Different mechanisms have been proposed to account for the transfer of information across generations, e.g., effects of environmental factors on the uterine environment, maternal care, and the epigenome ${ }^{14-16}$. Notably, while uterine changes and maternal care are likely to impact first-generation (F1) offspring, they are unable to account for changes observed in second- and third-generation offspring (F2 and F3, respectively), or for effects transmitted via the paternal lineage ${ }^{16-19}$. Epigenetic alterations in sperm were shown in several studies and may provide a biological mechanism for transmis$\operatorname{sion}^{20-25}$. In particular, changes in microRNA (miRNA) 
expression were proposed to occur after stress exposure in the parental generation and to account for behavioral changes in offspring ${ }^{26,20}$. Notably, the majority of studies on trans-generational stress effects have been performed in male rodents. Epigenetic changes in oocytes as mediators of inter- and trans-generational transmission remain poorly understood ${ }^{27}$. In particular, microRNA changes in oocytes have not been investigated as agents of inter- or trans-generational transmission of stress effects to date.

In a recent series of studies, we investigated the impact of mild, chronic pre-reproductive stress (PRS) to adolescent female rats on F1 and F2 offspring behavior, HPA axis function, prefrontal cortex (PFC) morphology, and gene expression patterns ${ }^{28-31}$. We observed changes in behavioral assays measuring fear and anxiety in F1 and maternally derived F2 offspring, changes in corticosterone (CORT) levels in exposed females and offspring, and alterations in expression of corticotropin-releasing factor receptor type 1 ( $C r h r 1)$, which plays a central role in the HPA axis response to stress ${ }^{28,29}$, in the PFC of exposed females and in the brain of their neonate offspring. Interestingly, Crhr1 levels in PRS-exposed female oocytes were substantially elevated, and were also increased in adult offspring PFC. Moreover, cortical Crhr1 expression depended on maternal as well as offspring exposure to stress.

Here, we first seek to determine whether PRS to adolescent female rats affects the expression of Crhr1targeting miRNAs in brain, blood, and oocytes. Relying on a miRNA database search and previous studies, and are some of a few miRNA molecules, which have been implicated on the stress response ${ }^{32-36}$, target $C r h r 1^{32,37-40}$, and are some of a few miRNA molecules expressed in oocytes ${ }^{41}$. Second, we ask whether maternal PRS affects Crhr1 and mir-34 expression in neonate F1 and F2 offspring, and alters expression patterns in animals that have been exposed to low- and high-stress conditions. Third, we inquire whether postPRS, pre-gestational pharmacological treatment can reverse the impact of PRS on offspring behavioral and molecular phenotypes. Specifically, we test the impact of the CRHR1 antagonist NBI-27914 (NBI) ${ }^{42}$ and the antidepressant fluoxetine (FLX), a selective serotonin reuptake inhibitor (SSRI) commonly prescribed to treat stress-related psychopathology in adolescence ${ }^{43,44}$ and previously shown to reverse the effects of chronic unpredictable stress in animal models ${ }^{45,46}$. Finally, since paternally derived offspring are less likely to be affected by the uterine environment and maternal care, we study PRS-induced changes in behavior and $\mathrm{mRNA} / \mathrm{miRNA}$ expression in F2 rats derived from F1 males, and compare them to our previous findings in maternally derived F2 offspring ${ }^{28,29}$.

\section{Methods}

Detailed methods and materials are provided in the Supplementary information (SI).

\section{Animals}

Adolescent female Sprague-Dawley rats and the adult males used for mating were purchased from Envigo (Jerusalem). The study was approved by the University of Haifa Committee on animal experimentation (294/13, $351 / 14,711 / 20)$. Animal care and experiments were performed in line with NIH guidelines and regulations.

\section{Experimental procedure}

The experimental timeline is outlined in Fig. 1 and described in detail in the Supplementary information (SI). Briefly, adolescent (P45) female rats (F0) were randomly assigned to control (C) or PRS groups. PRS rats were exposed to a 7-day chronic unpredictable stress (CUS) protocol $^{28-31,47}$. The 7-day procedure included (1) $15 \mathrm{~min}$ warm swim $\left(22^{\circ} \mathrm{C}\right),(2) 10 \mathrm{~min}$ cold swim $\left(15^{\circ} \mathrm{C}\right)$ followed by warming (3) $24 \mathrm{~h}$ food and water deprivation, (4) $24 \mathrm{~h}$ constant light, (5) 3 times $30 \mathrm{~min}$ on a raised platform at $30 \mathrm{~min}$ intervals, (6) electric shock $(10 \times 0.5 \mathrm{~mA} 1 \mathrm{~s}$ at $30 \mathrm{~s}$ intervals), (7) $24 \mathrm{~h}$ crowding ( 8 females in a cage $56 \times 35 \times$ $19 \mathrm{~cm}$ high) with constant light. Rats were then divided into 3 cohorts: P56 (Cohort 1), P66-73 (Cohort 2), and Drug Treatment (Cohort 3). Tissue samples were extracted from rats in Cohorts 1 and 2 on PND 56 and PND 66-73 (the mating period), respectively (see Fig. 1). $\mathrm{C}$ and PRS Cohort 3 rats received 5-7 days of intraperitoneal (i.p.) vehicle (VEH), NBI or FLX starting on P53. In $\mathrm{F} 1$, we examined male and female offspring of all six groups (F1-C/VEH, F1-C/NBI, F1-C/FLX, F1-PRS/VEH, F1-PRS/NBI, F1-PRS/FLX). To produce the F2 generation, we bred F1-C and F1-PRS behaviorally naïve males with naive females. In F2, we examined their male and female offspring (F2-C, F2-PRS). Experimental n's are depicted in figures.

\section{Behavior}

F0: Dams were tested for general locomotor abnormalities and novelty-induced anxiogenic behavior in the open field (OF). F1: Male and female adult progeny were randomly divided into low- and high-stress exposure. 'Low-stress' rats were tested in the OF followed $24 \mathrm{~h}$ later by Novel Object Recognition (NOR). 'High-stress' rats were tested in the elevated plus maze (EPM) followed $24 \mathrm{~h}$ later by the fear conditioning and extinction test. F2: Male and female adult progeny were tested in the OF, NOR, and social preference (SP) tests. Two weeks later, rats were tested in the EPM followed $24 \mathrm{~h}$ later by the fear conditioning and extinction test. Therefore, F2 progeny were all exposed to high-stress behavioral testing. Exclusion Criteria are detailed in 

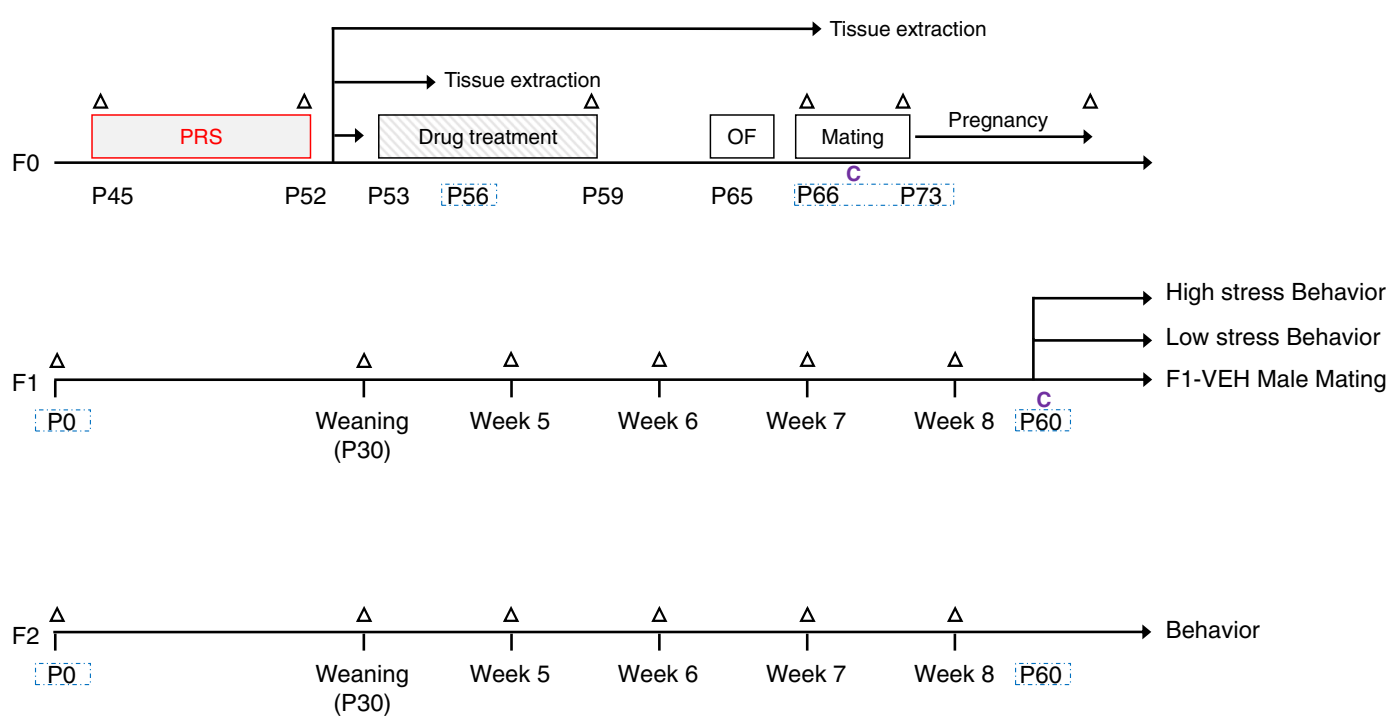

Fig. 1 Experimental procedure. Timeline of the experimental procedure in the parent (F0) and offspring (F1 and F2) generations. Triangle: determination of weight. Blue dashed rectangle: assessment of gene expression and/or miRNA levels. C: assessment of CORT levels.

Tables S1 and S2. Experimenters were blind to the group allocation during behavioral test performance.

\section{CORT quantification}

Blood was collected in F0 dams post-weaning, and in F1 adult behaviorally naïve males and females. Blood collection was performed in the morning, and CORT was quantified by ELISA as previously described ${ }^{28}$.

\section{mRNA and miRNA expression analysis}

We assessed mRNA and miRNA expression in oocytes and blood of F0 females, and in the AMY and PFC of F0 females and their neonate and adult F1 and F2 offspring. Dissections, RNA and miRNA extraction, cDNA preparation, and quantitative real-time PCR (qRT-PCR) were performed as described previously ${ }^{29,48,49}$ (see Table S3 for primer list). Fold-change values were calculated using the ddCt method ${ }^{50}$ relative to the housekeeping gene hypoxanthine phosphoribosyl transferase (HPRT; mRNA; brain), 18s (mRNA; oocytes), or U6-snRNA/RNU6 (miRNA), which were found to be expressed in similar levels across groups and tissue types.

\section{Statistical analyses}

The sample sizes of each experiment were determined based on our previous studies ${ }^{28,29}$. No randomization was performed. Data were analyzed with Student's two-sided $t$-tests, analysis of variance (ANOVA), repeated-measures ANOVA, multivariate analysis of variance (MANOVA), Pearson correlation coefficients, and the Chi-Square test of independence (see figure legends and SI for details) using SPSS 23 Statistics software (IBM, Chicago, IL). The least significant difference (LSD) test was used for posthoc comparisons when interactions were significant. Homogeneity of variance was confirmed with Levene's test for equality of variances.

\section{Results}

F0 dams and F1 neonates: basic attributes

Stress to adolescent female rats led to short-term weight loss and long-term weight gain (Fig. S1a), and decreased locomotor activity during the first minute in the OF; drug treatment had no effect on either measure (Fig. S1b). Examining F1 litter attributes, we found no PRS- or druginduced differences in litter size or in male/female pup ratio. However, maternal FLX or NBI decreased pup weight, and FLX treatment tended to increase pup mortality odds. Pup mortality was particularly high in the F1PRS/FLX group (Table S4).

\section{F0 dams, F1 and F2 neonates: mRNA and miRNA expression changes}

Raw dCt values for RT-PCR experiments are presented in Table S5. In F0 dams, we assessed mRNA and miRNA expression on P56 (4 days after stress) and during P66-73 (equivalent to the time of mating, see Fig. 1). In replication of our previous results ${ }^{29}$, we found an increase in Crhr1 mRNA in mPFC (Fig. 2a) of PRS-exposed female rats 4 days after exposure (P56). This increase was transient; by P66-73, Crhr1 expression was lower than control levels (Fig. 2b). In oocytes, Crhr1 mRNA was transiently elevated on P56, decreasing below control levels by 


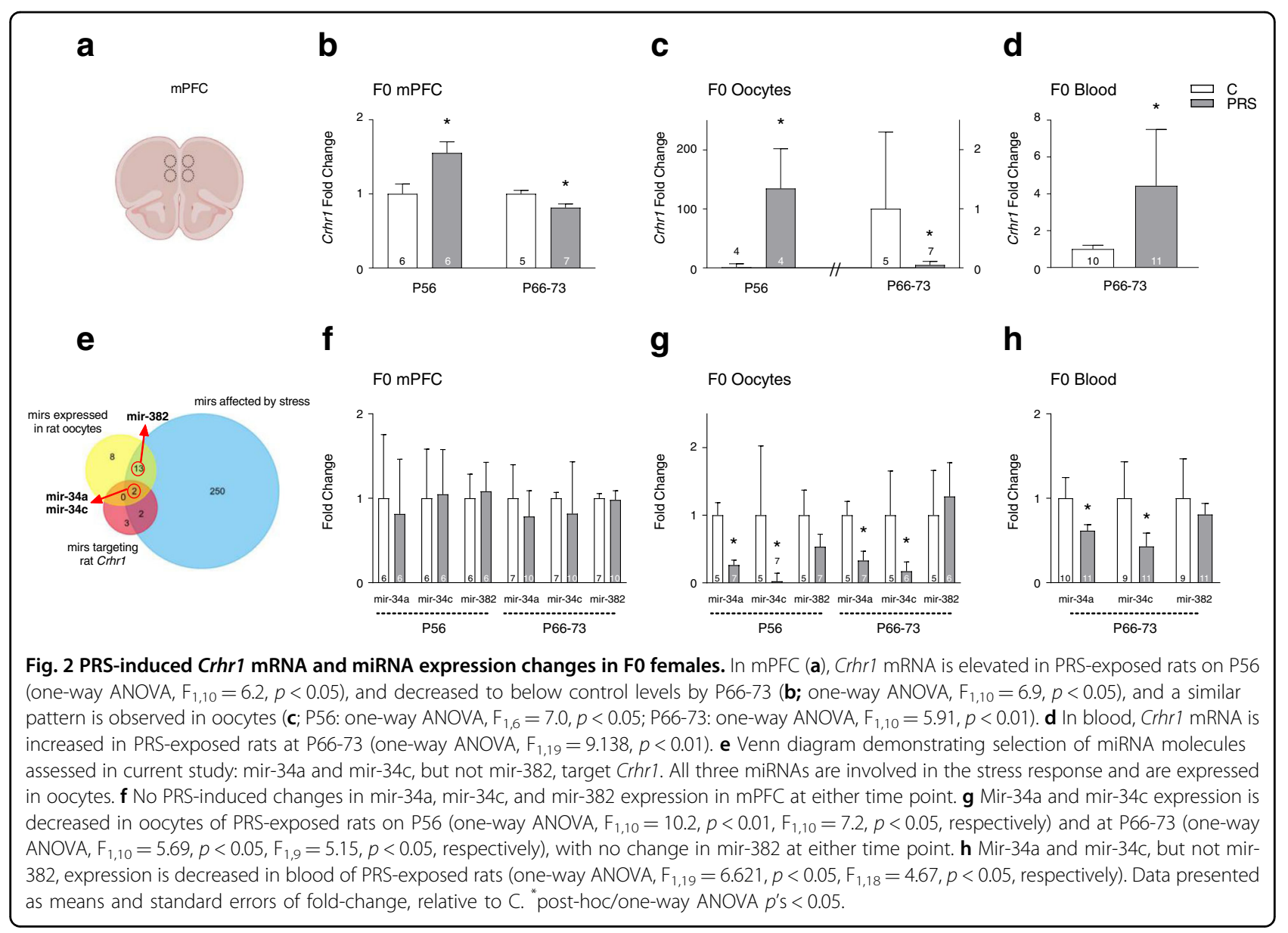

P66-73 (Fig. 2c). In blood, Crhr1 mRNA was elevated at P66-73 (Fig. 2d). PRS had no effect on Crhr1 expression in the AMY at either time point (Fig. S2a). Expression of CRH receptor type 2 (Crhr2) mRNA was not altered by PRS exposure in $\mathrm{mPFC}$ or AMY, and was undetected in oocytes or blood (not shown).

Relying on a miRNA database search, we focused our investigation on mir-34a, mir-34c, and mir-382. All three miRNA molecules are expressed in oocytes, the blood stream, and brain ${ }^{41}$ (confirmed by pilot studies in our lab), and participate in the stress response ${ }^{32-36}$; only mir34a and mir-34c, however, target $\mathrm{Crhr}^{32,37-40}$ (Fig. 2e). In mPFC, we found no PRS-induced changes in mir-34a and mir-34c at P56 or P66-73 and no changes in mir-382 expression at either time point (Fig. 2f). In the AMY, PRS had no effect on mir-34a or mir-382, but decreased mir34c expression at P56 (Fig. S2b). No change in mir-34a, mir-34c, and mir-382 was found in AMY at P66-73 (Fig. S2c). In oocytes, we found a 74\% PRS-induced decrease in mir-34a on P56 and a 67\% decrease at P66-73, with no change in mir-382 at either time point. Mir-34c decreased by $98 \%$ on P56 and $83 \%$ at P66-73 (Fig. 2g). A decrease in mir-34a and mir-34c, but no change in mir-382, was also observed in blood (Fig. 2h). Oocytic expression of additional non-Crhr1-targeting miRNAs (mir-137-3p, mir-137-5p, mir-203-3p, mir-203-5p, mir493-3p, and mir-493-5p) was not altered by PRS (Fig. S3).

We next analyzed PRS- and drug-induced changes in Crhr1 and mir-34a, mir-34c and mir-382 expression in PFC and AMY of F1 and F2 neonates. In F1 PFC (Fig. 3a), maternal PRS decreased Crhrl expression, and post-PRS treatment with the CRHR1 antagonist NBI or the SSRI FLX reversed this effect. Maternal FLX also increased Crhr1 in neonate offspring of Control dams. Mir-34a expression was increased in PFC of F1-PRS neonate offspring. A decrease in Crhr1 and an increase in mir-34a was similarly observed in neonate F2 offspring (Fig. 3b). In AMY, maternal PRS led to the opposite pattern of Crhr1 and mir-34a expression: it increased Crhr1 and decreased mir-34a expression in F1/VEH (Fig. 3c) and F2 neonates (Fig. 3d). In F1 AMY, post-PRS NBI treatment reversed PRS effects, and treatment of Control dams with NBI 
a

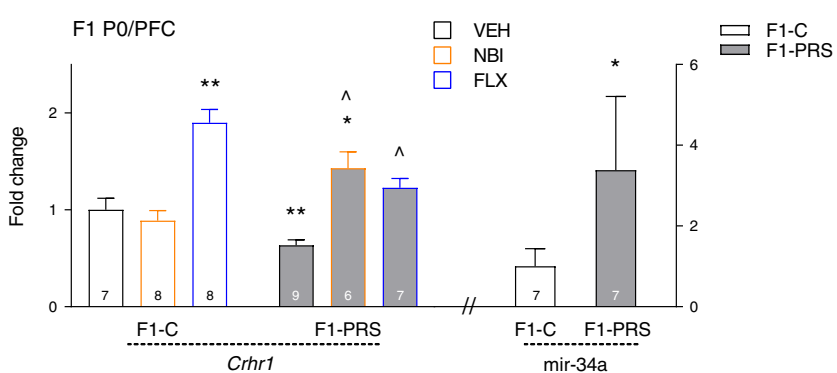

C

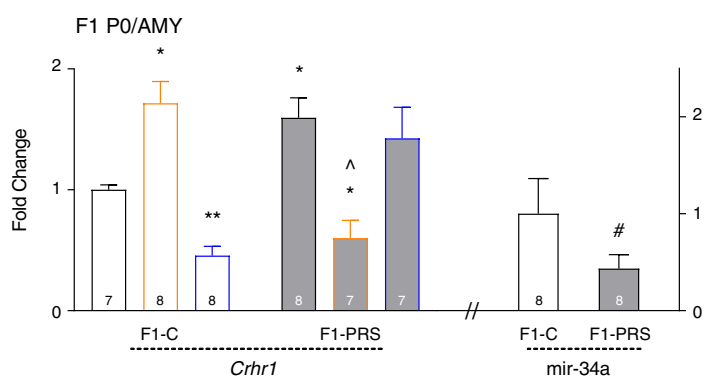

b

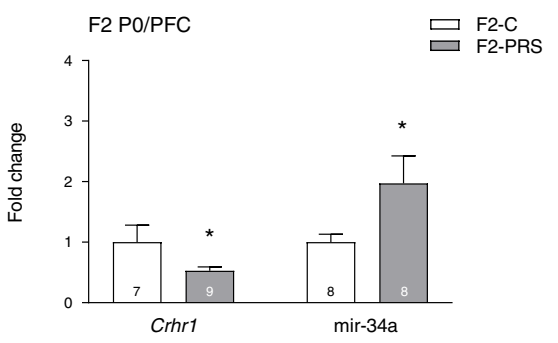

d

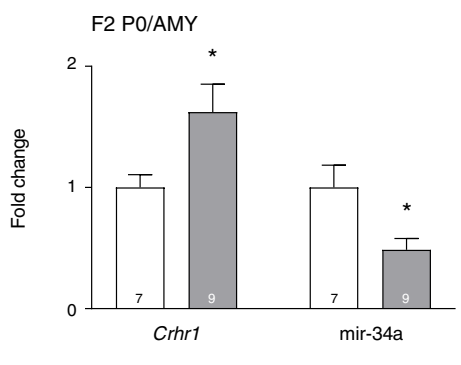

Fig. 3 PRS- and maternal drug treatment-induced gene/miRNA expression changes in neonate PFC and AMY of F1 and F2 offspring. a Maternal PRS decreases Crhr1 in PFC of neonate F1 offspring. This effect is normalized by post-PRS maternal treatment with FLX and NBI, which increases Crhr1 mRNA beyond control levels. Maternal FLX increases Crhr1 expression in F1-C ( $2 \times 3$ ANOVA, group $\times$ drug $\left.F_{2,39}=15.1, p<0.00001\right)$. Maternal PRS also increases mir-34a expression in F1-VEH PFC (one-way ANOVA, $F_{1,12}=5.6, p<0.05$ ). b As in F1-VEH animals, PRS decreases Crhr 1 mRNA (one-way ANOVA, $\left.F_{1,14}=8.1, p<0.05\right)$ and increases mir-34a expression $\left(F_{1,14}=7.7, p<0.05\right)$ in PFC of neonate F2 offspring. $\mathbf{c}$ Maternal PRS increases Crhr1 mRNA in AMY of neonate F1 offspring. This effect is normalized by post-PRS treatment with NBI, which decreases Crhr1 mRNA beyond control levels $\left(2 \times 3\right.$ ANOVA, group $\times$ drug $\left.F_{2,39}=29.2, p<0.00001\right)$. Maternal PRS also marginally decreases mir-34a expression in F1-VEH AMY (oneway ANOVA, $F_{1,14}=4.002, p=0.065$ ). d PRS increases Crhr1 mRNA (one-way ANOVA, $F_{1,14}=8.04, p<0.05$ ) and decreases mir-34a expression (oneway ANOVA, $F_{1,14}=6.5, p<0.05$ ) in AMY of neonate F2 offspring. Data presented as means and standard errors of fold-change relative to F1-CNEH (a, c) or F2-C (b, d). Post-hoc/one-way ANOVA ${ }^{\#} p<0.075,{ }^{*} p<0.05,{ }^{* *} p<0.001, \wedge p<0.05$, post-hoc relative to F1-PRS NEH.

increased, while FLX treatment decreased, Crhr1 mRNA. No change was found in mir-34c or mir-382 expression in F1 and F2 PFC or AMY (not shown).

\section{F1: weight gain in adolescence}

As can be seen in Table S6, both male and female F1PRS/FLX offspring gained less weight than rats in all other conditions.

\section{F1 adults: behavior changes under low- and high-stress conditions}

We tested adult F1 offspring behavior under low- and high-stress conditions. Initial $n$ 's, exclusion criteria, and final $n$ 's are summarized in Table S1. Male and female data were analyzed separately, since a main effect of sex and/or interactions with the sex variable were observed in all tests (see SI). In the low-stress cohort (Fig. 4a, b), male F1-PRS rats generally showed anxiogenic behavior: they spent less time in the center of the OF (Fig. 4a, left, center) and showed reduced overall exploration in the NOR Test Phase (Fig. 4b, center). Total locomotor activity and center latency in the OF, and object exploration in the Sample Phase of the NOR test were unaffected by maternal PRS or drug treatment. F1 female behavior in the OF was unaffected by maternal PRS or drug treatment (no differences in total locomotor activity, center latency, or center duration, Fig. 4a, right). In the NOR Sample Phase, female F1-PRS/ VEH explored more than F1-PRS/NBI and FLX $(2 \times 3$ ANOVA, drug $\left.\times \operatorname{sex} F_{2,146}=5.007, p<0.01\right)$. In the NOR Test Phase, F1-PRS/VEH females exhibited increased exploration compared to $\mathrm{F} 1-\mathrm{C} / \mathrm{VEH}$; maternal NBI and FLX treatment reversed this effect (Fig. 4b, right). Maternal drug treatment had no independent effects in either task. Novel object preference was present in all conditions and was unaffected by PRS or drug treatment in males and females (see SI).

In the high-stress cohort (Fig. 4c-f), maternal PRS led to decreased fear and increased risk-taking behavior in male offspring tested under high-stress conditions; in female offspring, the response was assay-dependent. In the EPM (Fig. 4c, left), male F1-PRS rats exhibited 


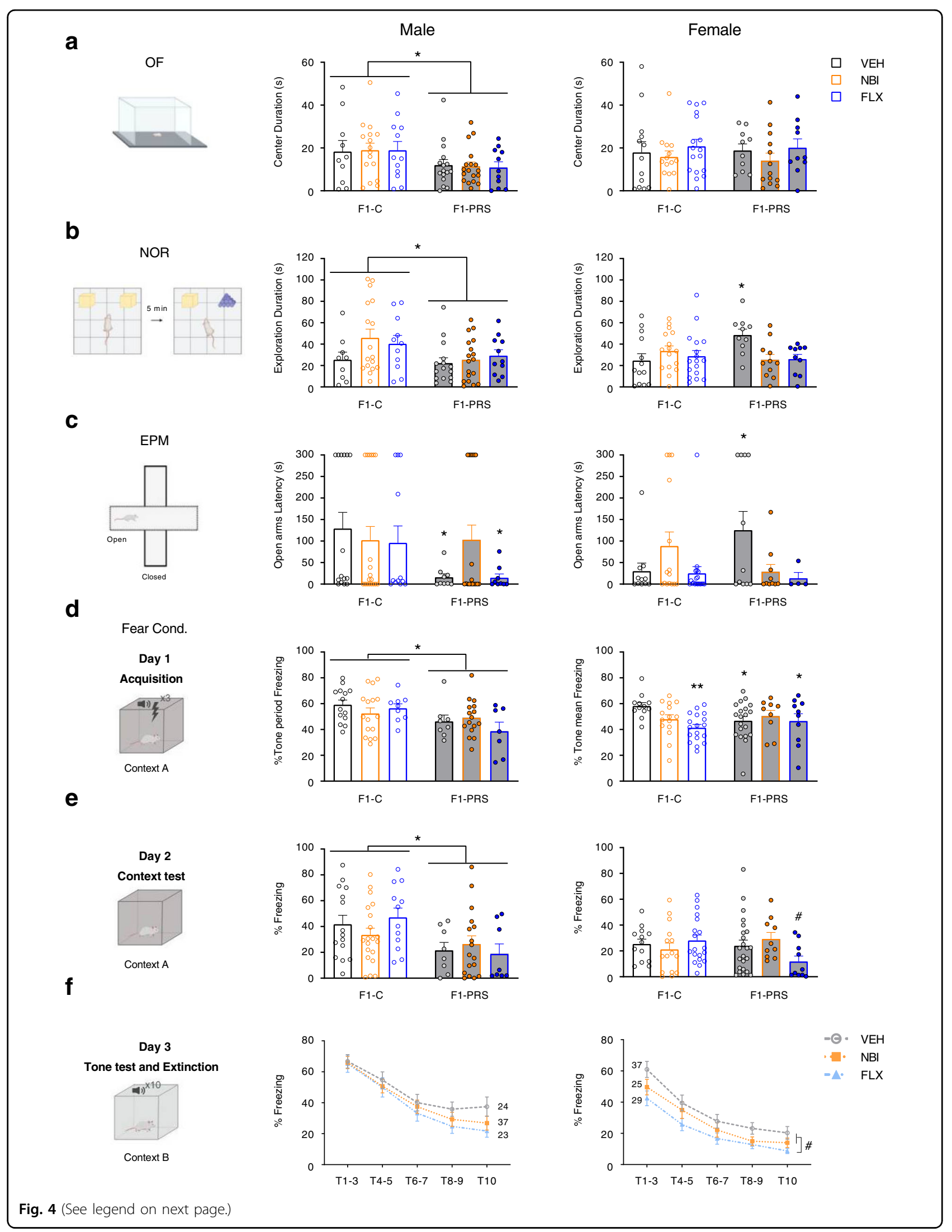


(see figure on previous page)

Fig. 4 Maternal PRS- and drug-induced changes in F1 offspring behavior under low- and high-stress testing conditions. a In the OF (left), male F1-PRS rats spend less time in the center of the OF compared to F1-C, regardless of maternal drug treatment (center; $2 \times 3$ ANOVA, group F $F_{1,83}$ $=7.6, p<0.01$ ), while female offspring are unaffected by maternal PRS or drug treatment (right). $\mathbf{b}$ In the NOR task (left), PRS decreases overall exploration time in males (center; $2 \times 3$ ANOVA, group, $F_{1,75}=4.45, p<0.05$ ), but increases it in females, and the latter effect is reversed by maternal treatment with either NBI or FLX (right; $2 \times 3$ ANOVA, group $\times$ drug $F_{2,71}=4.7, p<0.05$ ). $\mathbf{c}$ In the EPM (left), maternal PRS decreases open arm latency in males $\left(2 \times 3\right.$ ANOVA, group $\left.F_{176}=4.9, p<0.05\right)$, and subsequent NBI treatment reverses this effect (center; one-way ANOVA on F1-CNEH and F1PRS groups, $\left.F_{3,47}=2.9, p<0.05\right)$. In female offspring, maternal PRS increases latency, and subsequent treatment with either NBI or FLX reverses this effect (right; one-way ANOVA on F1-CNEH and F1-PRS groups, $F_{3,32}=2.9, p<0.05$ ). d On the Acquisition Day (Day 1 ) of the fear conditioning/ extinction task, (left), maternal PRS decreases freezing during the tone period in male offspring ( $2 \times 3$ ANOVA, group $\left.F_{1,64}=9.3, p<0.01\right)$, and subsequent drug treatment had no effect (center). In female offspring (right), we observe an interaction that approaches significance between group and drug treatment $\left(2 \times 3\right.$ ANOVA, $\left.F_{2,78}=3.1, p=0.052\right)$, so that maternal PRS and/or FLX decreased freezing during tone presentation. e During the Context Test (Day 2) (left), maternal PRS decreases freezing in male offspring, and subsequent drug treatment has no effect (center; $2 \times 3$ ANOVA, group $\left.F_{1,74}=10.4, p<0.01\right)$. In female offspring (right), only PRS followed by FLX marginally decreases freezing $\left(2 \times 3\right.$ ANOVA, group $\times$ drug $F_{2,84}=$ 3.0, $p=0.055$ ). $\mathbf{f}$ During the Tone Test/Extinction (Day 3), gradual extinction of fear is observed in male offspring (repeated-measures ANOVA, tone $F_{4.4,343.2}=25.7, p<0.001$ ), but maternal PRS or drug treatment have no effect on freezing during tones $1-3$ (Tone Test) or the subsequent 7 tones (Extinction; center). In female offspring (right), freezing is extinguished over time (repeated-measures ANOVA, tone $F_{4.3,370}=20.3, p<0.0001$ ), maternal FLX treatment decreases freezing during tones $1-3$ (repeated-measures ANOVA, drug $F_{2,85}=4.1, p<0.05$ ), and marginally decreases freezing in tones 4-10 (repeated-measures ANOVA, drug $F_{2,85}=3.0, p=0.055$ ) regardless of maternal PRS exposure. Data presented as individual values, with bars and whiskers representing means and standard errors, respectively (a-e), or as means and standard errors $(\mathbf{f}) . \# p<0.075,{ }^{*} p<0.05$, ${ }^{* *} p<0.001$, post-hoc relative to F1-CNEH.

decreased latency to open arms, and this effect was reversed by maternal NBI, but not FLX, treatment (Fig. $4 c$, center). Male F1-PRS rats exhibited increased frequency $\left(\mathrm{F}_{1,76}=7.1, \quad p<0.01\right)$ and a trend towards increased duration in the open arms of the EPM $\left(\mathrm{F}_{1,76}=\right.$ $3.4, p=0.07)$. Latency to open arms was increased in female F1-PRS/VEH rats, and this effect was absent if maternal PRS was followed by NBI or FLX (Fig. 4c, right). No differences in open arm frequency and duration, or closed arm latency, frequency and duration were found (data not shown).

In the same rats, we measured freezing during delay fear conditioning (Day 1), contextual and cued recall tests (Days 2 and 3, respectively), and cue extinction (Days 3-5). Data from some rats were excluded because of stereotypic behavior, which was not influenced by maternal PRS or drug exposure (see exclusion criteria in Table S1). On Day 1 (Fig. 4d, left), freezing during tone presentation increased gradually in male and female offspring, indicating intact conditioning to tone (repeated-measures ANOVA, $\quad \mathrm{F}_{2,128}=208.1, \quad p<0.0001$, $\mathrm{F}_{2,156}=414, p<0.0001$, respectively). Male F1-PRS rats froze less than $\mathrm{F} 1-\mathrm{C}$ controls during the entire tone period and maternal drug treatment did not reverse this effect (Fig. 4d, center). In females, average freezing during the 3 tone presentations, but not the entire tone period, was reduced by maternal PRS, as well as by maternal FLX administration (Fig. 4d, right). On Day 2 (Fig. 4e, left), male F1-PRS rats froze less than F1-C controls (Fig. 4e, center); in females, F1-PRS/FLX rats tended to show the lowest freezing levels overall (Fig. 4e, right). On Day 3 (Fig. 4f, left), we found increased freezing during the average of the first 3 tones compared to the pre-tone period in males $\left(\mathrm{F}_{1,78}=288.6, p<0.001\right)$ and females $\left(\mathrm{F}_{1,85}=240.0, p<0.0001\right)$, and gradual extinction of fear during the 7 subsequent tones in males (tone, $\mathrm{F}_{4.4,343.2}=25.7, p<0.001$ ). Maternal PRS or drug treatment had no effect on freezing during tone recall (tones 1-3) or extinction (tones 4-10) in males (Fig. 4f, center). Female F1-FLX rats froze less than F1VEH and F1-NBI rats, regardless of maternal PRS, during tone recall and extinction (Fig. 4f, right). No maternal PRS effects were observed on Day 4 or 5 (see SI).

\section{F2 neonates: basic attributes}

Naïve female rats mated with F1-PRS males gained more weight during pregnancy and weighed more than those mated with F1-C males 30 days after parturition (Table S7), although there were no differences in litter size or pup weight (Table S8).

\section{F2 adults: behavior}

Behavioral analysis in adult F2 offspring revealed that in most tasks, grandmaternal PRS affected female, but not male, offspring. In the OF, PRS induced higher locomotion during the first $5 \mathrm{~min}$ in F2 female offspring; no differences were found in males (Fig. 5a). Analysis of the latency to enter the center(s) revealed that F2-PRS females entered the center of the OF sooner than their F2-C counterparts. Again, no differences were observed in males (Fig. 5b). No differences were found in males or females in the time spent in the center of the OF (not shown). In the NOR assay, grandmaternal PRS had no 


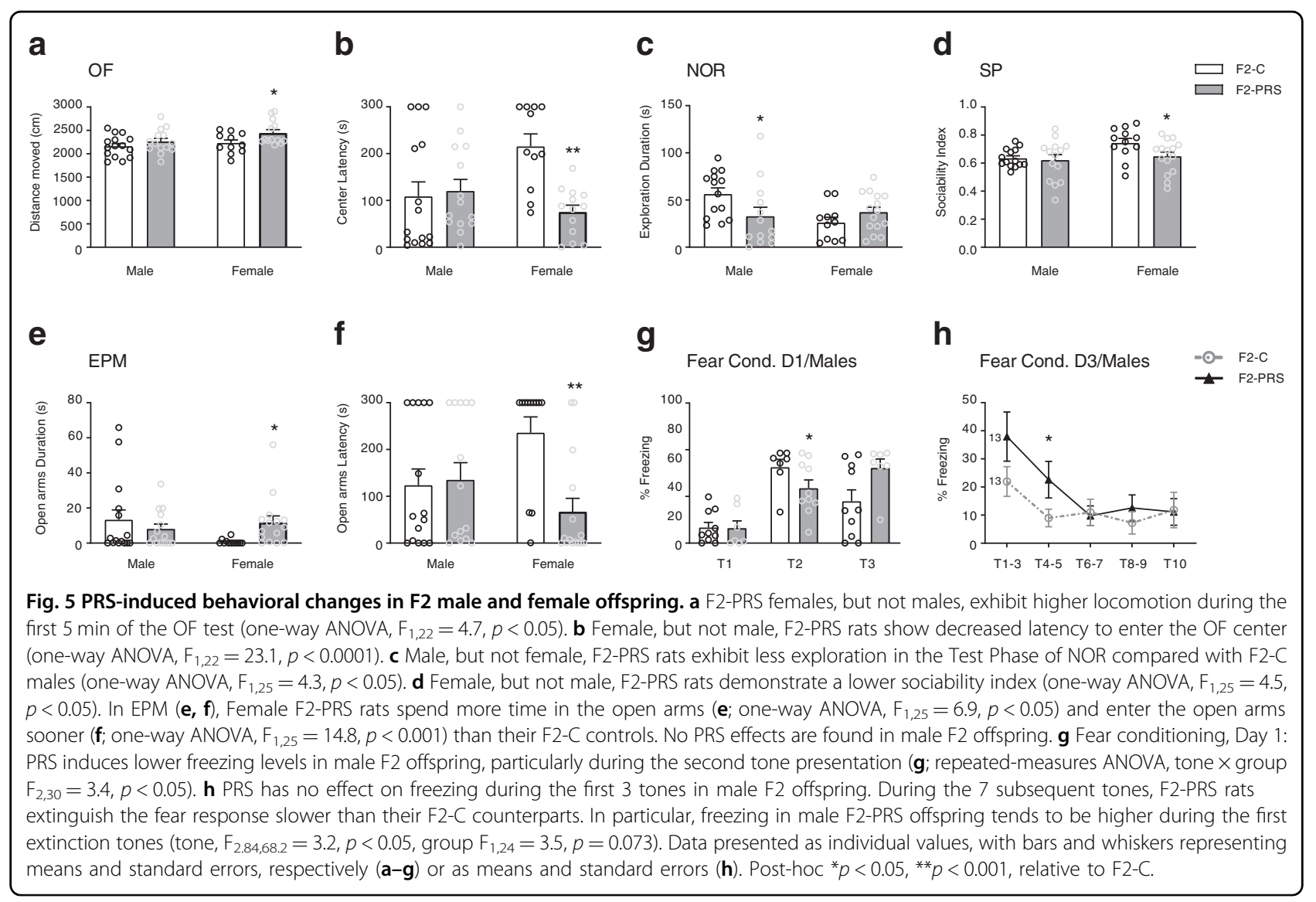

effect on exploration time or the latency to approach either object during the Sample Phase (data not shown). In the Test Phase (Fig. 5c), male F2-PRS explored less than F2-C. Notably female F2-C also explored less than male controls. Neither F2-C nor F2-PRS rats showed a preference towards the novel stimulus (not shown). In the SP task, female F2-PRS rats exhibited lower scores in the sociability index compared to F2-C; no differences were found in males (Fig. 5d).

In the EPM, female F2-PRS rats spent more time in the open arms (Fig. 5e), and entered the open arms sooner (Fig. 5f) and more frequently $\left(\mathrm{F}_{1,25}=12.3 p<0.01\right)$ compared with their F2-C controls; no differences were found in males. Notably, F2-C females spent significantly less time and showed greater latency to enter the open arms than F2-C males (Fig. 5e, f).

In the fear conditioning and extinction task, male F2PRS rats exhibited high freezing levels on Day 1 compared with F2-C controls, particularly during the second tone presentation (Fig. 5g). In females, freezing was not affected by grandmaternal PRS (data not shown). Grandmaternal PRS had no effect on freezing to context (Day 2) or during the first 3 tones (Day 3), in either males or females (two-way ANOVAs; not shown). Analysis of the 7 subsequent tones on Day 3 revealed a tendency for slower extinction of the fear response in male F2-PRS rats (Fig. 5h). No significant PRS effects were found in females (not shown). On Day 4 and 5, we found low freezing levels $(<15 \%)$, and no extinction effects in either males or females (not shown). Rat exclusion details and further analysis details are presented in Table S2 and the SI.

\section{F1 and F2 adults: mRNA and miRNA expression changes}

In agreement with our previous observations ${ }^{28,29}$, direct exposure to low- and high-stress testing conditions interacted with maternal stress exposure in its impact on F1 Crhr1 expression (Fig. 6a, b), with different effects in male and female rats. In males, high-stress testing conditions increased, but maternal PRS decreased, Crhr1 mRNA expression (Fig. 6a). In females, high-stress testing increased Crhr1 mRNA. Maternal PRS increased Crhr1 expression in low-stress, but decreased it in high-stress females (Fig. 6b). We asked whether increased Crhr1 expression would be accompanied by decreased mir-34a expression, as observed in germline cells and neonates, but changes in mir-34a seemed to vary independently of 
a

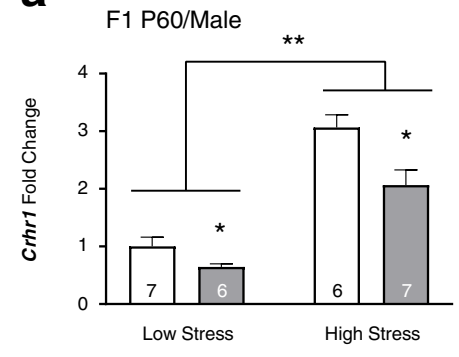

C

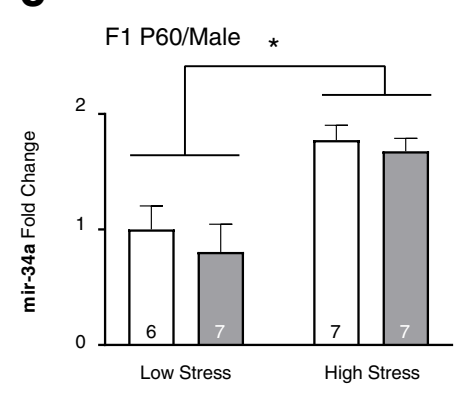

e

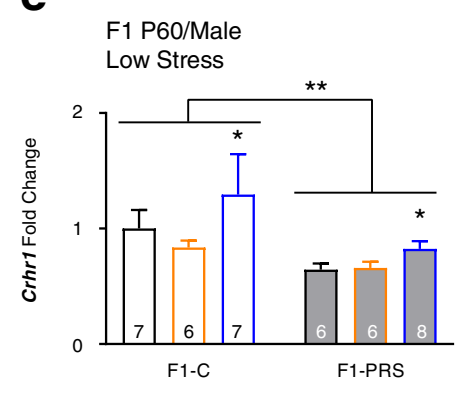

9

F1 P60/Male High Stress

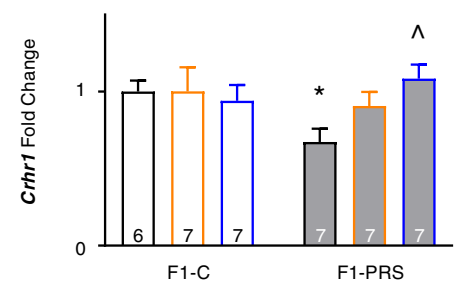

i

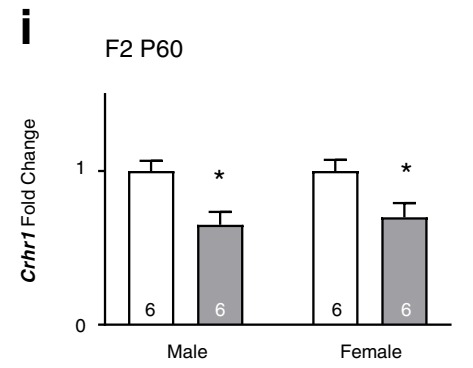

b
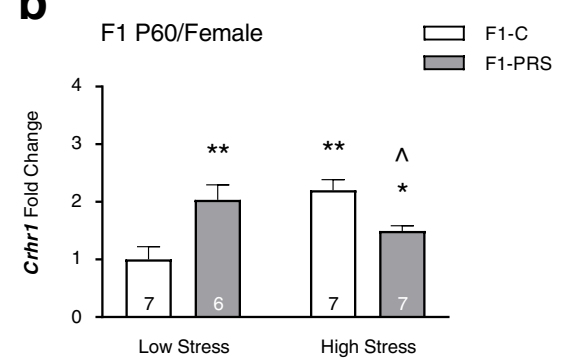

d

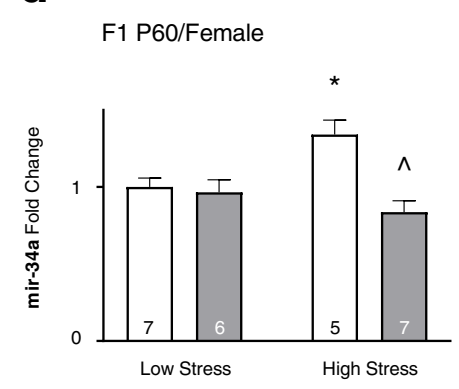

f

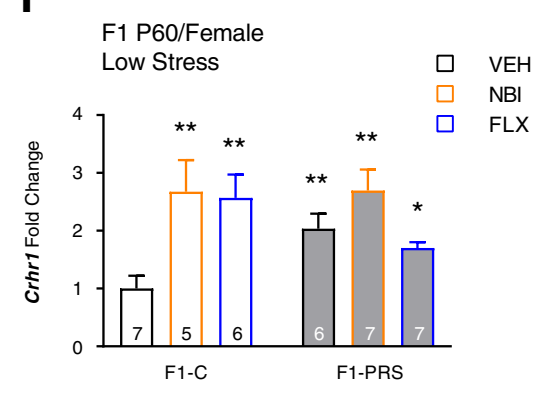

h F1 P60/Female High Stress
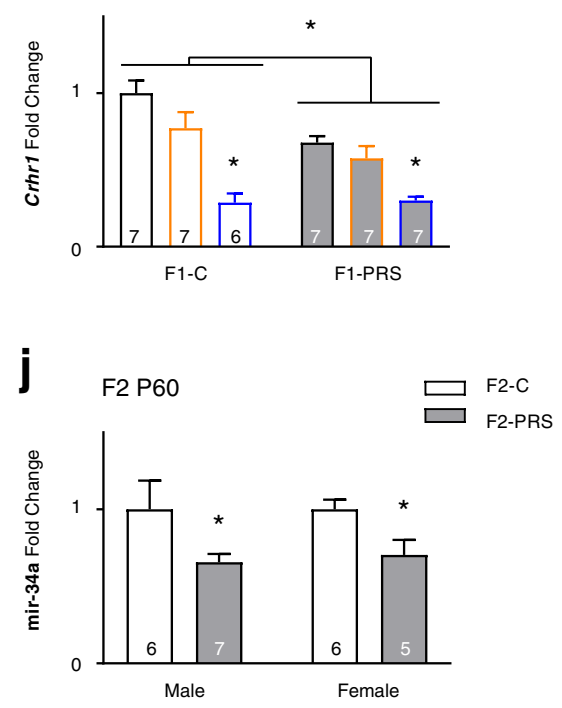

Fig. 6 (See legend on next page.) 
(see figure on previous page)

Fig. 6 Effects of maternal PRS and drug treatment, and offspring exposure to behavioral stress on Crhr1 and mir-34a expression in mPFC of adult F1 and F2 offspring. a In F1-VEH males, direct exposure to high-stress testing conditions increases, whereas maternal PRS decreases, Crhr 1 expression $\left(2 \times 2\right.$ ANOVA, cohort $F_{1,22}=117.08, p<0.00001$; group $\left.F_{1,22}=15.6, p<0.001\right)$. b In F1-VEH females, high-stress test exposure increases Crhrl expression, whereas maternal PRS increases Crhrl expression in low-stress but decreases it in high-stress rats (group $\times$ cohort $F_{1,23}=19.2, p<$ 0.001). c In F1-VEH males, high-stress test exposure increases mir-34a expression $\left(2 \times 2\right.$ ANOVA, cohort $\left.F_{1,23}=10.55, p<0.01\right)$. d In F1-VEH females, high-stress test exposure increases mir-34a expression in F1-C rats, and maternal PRS decreases expression in high-stress rats only $(2 \times 2$ ANOVA, group $\times$ cohort $\left.F_{1,21}=7.06, p<0.05\right)$. e In low-stress F1 males, PRS leads to a decrease in Crhr1, and maternal FLX treatment increases Crhr 1 expression ( $2 \times 3$ ANOVA, group $F_{1,34}=16.5, p<0.001$; drug $\left.F_{2,34}=4.8, p<0.05\right)$. $\mathbf{f}$ In low-stress $F 1$ females, maternal PRS and/or drug treatment increase Crhr1 expression $\left(2 \times 3\right.$ ANOVA, group $\times$ drug $\left.F_{2,32}=7.9, p<0.01\right) . \mathbf{g}$ In high-stress F1 males, maternal PRS decreases Crhrl expression, and this is reversed by maternal FLX treatment (group $\times$ drug $F_{2,35}=3.1, p=0.059$ ). $\mathbf{h}$ In high-stress F1 females, maternal PRS and/or FLX treatment decreases Crhr1 mRNA (group $F_{1,35}=4.9, p<0.05$; drug $F_{2,35}=42.2, p<0.001$ ). In F2 offspring, PRS decreases Crhr1 (i) and mir-34a (j) expression in male and female offspring (1-way ANOVAs Crhr1: $\mathrm{M} \mathrm{F}_{1,10}=5.43, p<0.05 ; \mathrm{FF}_{1,10}=5.6, p<0.05 ;$ mir-34a: $\mathrm{M} \mathrm{F}_{1,11}=5.42, p<0.05 ; \mathrm{FF}_{1,9}=6.3, p<0.05$ ). Data presented as means and standard errors of fold-change. ${ }^{*} p<0.05,{ }^{* *} p<0.001$, post-hoc or main effects relative to C-VEH controls. $\wedge p<0.05$, post-hoc relative to high-stress F1-VEH or F1-PRS/NEH.

Crhr1 in adults. Mir-34a expression was increased in males exposed to high-stress conditions, regardless of maternal PRS (Fig. 6c). In females, exposure to high-stress conditions increased mir-34a expression in offspring of Control dams (Fig. 6d), and maternal PRS decreased expression in high-stress, but not in low-stress, offspring.

Maternal drug treatment reversed the impact of maternal PRS on Crhrl expression in male offspring exposed to highstress, but not low-stress, conditions, and FLX had independent effects on low-stress offspring (Fig. 6e, g). In female F1 offspring, maternal PRS or drug treatment led to increased expression under low-stress conditions (Fig. 6f). Under high-stress conditions, maternal PRS or FLX decreased the Crhrl expression whereas NBI had no effect (Fig. 6h). Examining the impact of maternal NBI or FLX treatment on serum CORT in control and PRS-exposed females and their offspring, we found that maternal NBI treatment, which reversed the impact of PRS on serum CORT in F0, also reversed the 3.5-fold PRS-induced increase in CORT observed in behaviorally naive F1 offspring $^{28}$, whereas FLX administration had the opposite effect and increased CORT by $>8$ fold (Table S9).

In F2, we assessed rats that underwent a high-stress testing battery. As in the F1 high-stress cohort, PRS decreased Crhr1 mRNA expression (Fig. 6i). It also significantly decreased mir-34a expression in both male and female F2 offspring (Fig. 6j).

We examined maternal PRS-induced fluctuations in the expression of Crhr2 in adult F1 offspring tested in low-stress and high-stress conditions (Fig. S4). We found that Crhr1 and $C r h r 2$ were negatively correlated high-stress F1-PRS rats $(n=12, r=-.842, p=0.001)$; there was no correlation in low-stress rats or in F1-C offspring. In male F1 offspring, $\mathrm{Crhr} 2$ expression was increased by both maternal PRS and exposure to high-stress conditions, but was normalized to control levels in high-stress/F1-PRS offspring (Fig. S4a). In female offspring, the opposite pattern was observed: $\mathrm{Crhr} 2$ expression was decreased by both maternal PRS and highstress testing conditions, but high-stress/F1-PRS females showed normal Crhr2 expression (Fig. S4b). Drug treatment normalized aberrations in Crhr2 expression in low-stress males (Fig. S4c) and females (Fig. S4d), as well as in highstress males (Fig. S4e). In high-stress females, maternal drug treatment had no effect (Fig. S4f). In F2 offspring (exposed to high-stress conditions), Crhr2 was decreased in male, but not in female, offspring (Fig. S5).

\section{Discussion}

Chronic, unpredictable stress in adolescence, prior to gestation, leaves molecular and behavioral footprints in subsequent generations. Here, we demonstrate for the first time that exposure of female rats to PRS induces changes in blood and oocyte miRNA expression, and similar changes in miRNA expression in the PFC of neonate F1 and F2 offspring. Furthermore, we find that some of the effects of PRS in offspring can be reversed by maternal pharmacological interventions. Finally, we show that FLX treatment in adolescence impacts on reproductive health and offspring stress susceptibility.

Four days after stress exposure, we observe an increase in Crhr1 mRNA in PRS-exposed dams, in line with our previous findings ${ }^{28,29}$. In parallel, the expression of mir34a and mir-34c, which target Crhrl and block its transcription $^{32,37,51}$, is reduced in oocytes of PRS rats while the expression of non-Crhr1-targeting miRNAs is unaltered (Figs. 2g and S3). The mir-34 family of microRNA molecules are associated with the stress response $^{52}$ as well as with longevity and brain aging ${ }^{53}$. Specifically, alterations in mir-34 are associated with resilience under stress conditions ${ }^{51}$. This is the first report of stress-induced alterations in rat oocyte miRNA expression. miRNA expression changes have been detected in mouse sperm ${ }^{20,21,26,54}$, and were suggested to provide a mechanism for epigenetic germline 
inheritance in mammals ${ }^{55-57}$. Oocyte miRNAs may play a similar role, and transmit not only genomic but also maternal epigenomic information across generations ${ }^{27}$. Notably, the increase in oocytic Crhrl is transient, while the decrease in mir-34 remains low at the time of mating. The mechanism underlying the translation of behavioral stress to altered expression of germline miRNA molecules is unknown, and may involve HPA axis activation ${ }^{58-61}$ affecting blood-germline cell communications ${ }^{20,62-64}$. In support of this possibility, our previous studies point to increased CORT levels, and our present findings show increased Crhrl mRNA and decreased miR-34a in blood of stress-exposed F0 females (Fig. 2d, h). Notably, Crhr2 (which is also targeted by mir-34) was not detected in blood or oocytes.

Changes in mir-34a and mir-34c expression in oocytes of stress-exposed dams may be directly or indirectly responsible for the reduction in Crhr1 mRNA observed in the PFC of their neonate F1 and F2 offspring (Fig. 3a, b). Previous studies have shown that mir-34c reduces the responsiveness of cells to CRF in vitro ${ }^{32}$. Thus, changes in germline mir-34 expression could alter sensitivity to HPA axis signals in utero and impact cortical development in F1. Alternatively, reduced Crhr1 at birth could stem from abnormal in utero cortical development. Future studies should examine whether miRNA expression patterns are also altered in sperm of F1-PRS males, which in the present study were mated with naïve females to produce F2 offspring. Such alterations could account for Crhr1 expression abnormalities in the brain of neonate F2 offspring.

The reduction in cortical Crhr1 expression in F1 and F2 neonate brain was accompanied by an increase in mir-34a expression. This increase could substantially hinder cortical maturation; overexpression of mir-34a in cortical neurons was found to increase cellular vulnerability $^{65}$. In our model, overexpression could lead to malprogramming of the HPA axis and account for our previously reported abnormalities in adult PFC morphology in F1-PRS rats $^{30}$. It should be noted that an inverse pattern of changes (i.e., increased Crhrl and decreased mir-34a, similar to patterns observed in oocytes and blood) was observed in neonate AMY in F1 and F2; this observation supports previous evidence for different developmental patterns of the HPA axis in PFC and $\mathrm{AMY}^{66,67}$.

In contrast with neonates, stress-induced changes in Crhr1 mRNA in brain of F0, F1, and F2 adult animals of all three generations were not paralleled by mir-34a expression changes (Figs. 2 and 6). Possibly, stressinduced changes in miRNA expression could be quick and transient, returning to normal levels by P56. Alternatively, changes in mir-34a and Crhrl in blood and oocytes as well as in neonate brain may be a specific marker of the inter-generational transfer of stress effects, and not stress per se. Generally speaking, phenotypes observed in adult offspring could be the consequence of stress-induced alterations in maternal care. Notably, such alterations are less likely to account for changes in F2, since F2 offspring were derived from male F1 offspring and naïve females.

Our behavioral assays show that male F1-PRS rats showed decreased center exploration in the OF and reduced novelty exploration in the NOR task (Fig. 4a, b), along with increased exploration of the EPM open arms and less freezing during acquisition of the fear response (Fig. $4 \mathrm{c}-\mathrm{f}$ ). These phenotypes are consistent with the idea that maternal PRS induces in male offspring a more 'cautious' phenotype under benign circumstances, but 'inoculates' them against acute stress or perceived danger. This is in line with previous studies in humans and animals, which show that exposure to stress in early life or adolescence can result in stress susceptibility and also in resilience, depending on, e.g., type of stressor or offspring $\operatorname{sex}^{6,7,29,67-70}$. Further investigations into the advantages and disadvantages of parental stress in various offspring environments are required for in-depth understanding of stress susceptibility and resilience.

In agreement with our previous studies, Crhr1 expression in adult mPFC depends on maternal as well as offspring exposure to stress (Fig. 6). Crhrl expression was higher in offspring exposed to stressful testing conditions compared to benign behavioral tasks. Maternal exposure to stress, however, affected offspring in a sex-dependent manner, decreasing Crhr1 in males and increasing it in females (Fig. 6). In parallel, maternal PRS mitigated anxiogenic behavior in males, but exacerbated it in females (Fig. 4c-f). Interestingly, expression of Crhr2, which is not targeted by mir-34a or mir-34c, was negatively correlated with Crhrl expression in F1-PRS offspring and was affected by maternal PRS, sometimes in a pattern opposite to what we observed with Crhr1 (e.g., Figs. 6b and S4b). However, changes in Crhr2 were not paralleled by behavioral alterations. Furthermore, $\mathrm{Crhr} 2$ levels in F0 were unaltered by stress, although this effect should be examined at additional time points. Crhr1 expression may thus be more closely associated with changes in anxiogenic behavior, and expression of Crhr1 and Crhr2 in offspring is likely to be mediated by different mechanisms.

Some of the behavioral and molecular findings in adult rats agree with our previous findings ${ }^{28,29,49,71}$, while others differ. This may be due to differences in the order and identity of the behavioral assays, which could have affected behavioral and, well as, gene expression profiles. For example, the 'low-stress' behavioral cohort in 
the present study included the OF, NOR, and SP tests, while in our previous study 'low-stress' rats were tested in OF only. The NOR and SP tasks involve novelty exposure, which impacts gene expression patterns in the $\mathrm{mPFC}^{72}$ and engages the HPA axis ${ }^{73}$; this may explain differences in cortical Crhrl expression patterns between the current study and our previous investigations. Similarly, differences in fear acquisition between our present and past findings may be accounted for by previous exposure to a fear-eliciting environment: in the present study, rats were first exposed to the high-stress EPM assay, whereas previously they were tested in the fear conditioning assay alone. These differences highlight the complex interactions between parental and direct exposure to environmental factors, e.g., novelty or stress, and merit further investigation. Sex differences in both F1 and F2 offspring are in line with our previous experiments and a plethora of other studies (e.g., ${ }^{74}$ ), and could result from differential interference of stress with sex hormone signaling, or from sex-dependent differences in epigenetic regulation ${ }^{75}$.

CRHR1 antagonists were previously shown to reverse the sequelae of early developmental or adult stress ${ }^{76,77}$, CRF administration ${ }^{78}$, and mir-34 knockout ${ }^{37}$. Here, maternal post-PRS subchronic treatment with the CRHR1 antagonist NBI reverses PRS-induced changes in Crhr1 expression in neonate offspring, as well as abnormalities in serum CORT and behavior in adult progeny (Figs. 3, 4c-f, and Table S9). This is the first demonstration of combined stress and drug effects in adult offspring of exposed rats, and further supports the role played by Crhr1 elevation in the transmission of stress effects.

Surprisingly, subchronic administration of FLX to adolescent females, prior to gestation, increases pup mortality and reduces offspring weight from birth to early adulthood, particularly when followed by stress exposure. Administration of high-dose FLX and other SSRIs during gestation and lactation was previously demonstrated to increase neonatal mortality and decrease birth weight in rodents ${ }^{79-85}$. In our study, FLX was administered pre-gestationally and at a relatively low dose. FLX is commonly administered during adolescence, a time period of heightened stress sensitivity ${ }^{86}$. However, the impact of adolescent pre-gestational FLX use on neonatal viability and early brain development has scarcely been explored ${ }^{87}$.

In adult offspring, maternal FLX treatment exacerbated the effect of maternal PRS on serum CORT, but had some beneficial effects on behavior in offspring of stress-naïve as well as PRS rats (Fig. 4 and Table S9). The latter findings are in agreement with prior rodent literature, where FLX reversed stress-induced anxiogenic and depressive symptoms as well as memory impairments in offspring ${ }^{88,89}$. The mechanism/s underlying the effects of FLX in neonate and adult offspring remain to be determined, and may involve an ongoing effect of FLX and its active metabolite, norfluoxetine, on the developing fetus despite discontinuation of FLX a week prior to mating ${ }^{90,91}$. FLX could also affect neonate and adult offspring phenotypes by its indirect effects on the blood-brain barrier ${ }^{92}$ and the HPA axis ${ }^{93-99}$. Another interesting possibility is that FLX treatment affects the quality of maternal care ${ }^{100}$; this possibility should be examined in future studies.

As in our previous study ${ }^{28}$, we observed Crhr1, CORT, and behavior changes in adult PRS F2 offspring. Some behavioral effects in the present study were similar in F1 and F2, e.g., NOR exploration times decreased in both F1 and F2 males (Figs. 4b and 5c) and EPM abnormalities in paternally derived F2-PRS females were similar to those found in F1-PRS males (Figs. 4c and 5f). A comparison of the current study with our previous investigation ${ }^{28}$, where an identical experimental design was used but F2 offspring were derived from $\mathrm{F} 1$ females, reveals that maternal and paternal transmission produce different behavioral and molecular phenotypes in offspring. In general, transmission via the paternal lineage leads to behavioral alterations in female offspring, whereas transmission via the maternal lineage affects offspring of both sexes.

Interestingly, naive females mated with PRS F1 males gained significantly more weight during pregnancy. This may reflect emotional transfer effects ${ }^{101,102}$, and points to an altered in utero environment affecting F2 phenotypes. Germline cells of F1 offspring, as well as maternal behavior in F1, should also be examined to clarify the relative roles of social and epigenetic mechanisms in transmission.

In sum, our findings point to epigenetic mechanisms as a putative mediator of stress transmission across generations (see summary Fig. 7). Clearly, these mechanisms may interact with social factors, i.e., maternal care, which were also shown to impact similar stress-related pathways ${ }^{103,104}$. This interaction should be more extensively investigated in future studies. Furthermore, the findings of the present investigation indicate that pharmacological intervention may be effective in reversing some of the effects of stress across generations, while having its own impact on some measures. Finally, this study highlights the importance of studying stress transmission, susceptibility, and resilience in both genders, since the impact of adversity and mechanisms of transmission differ significantly between males and females. 


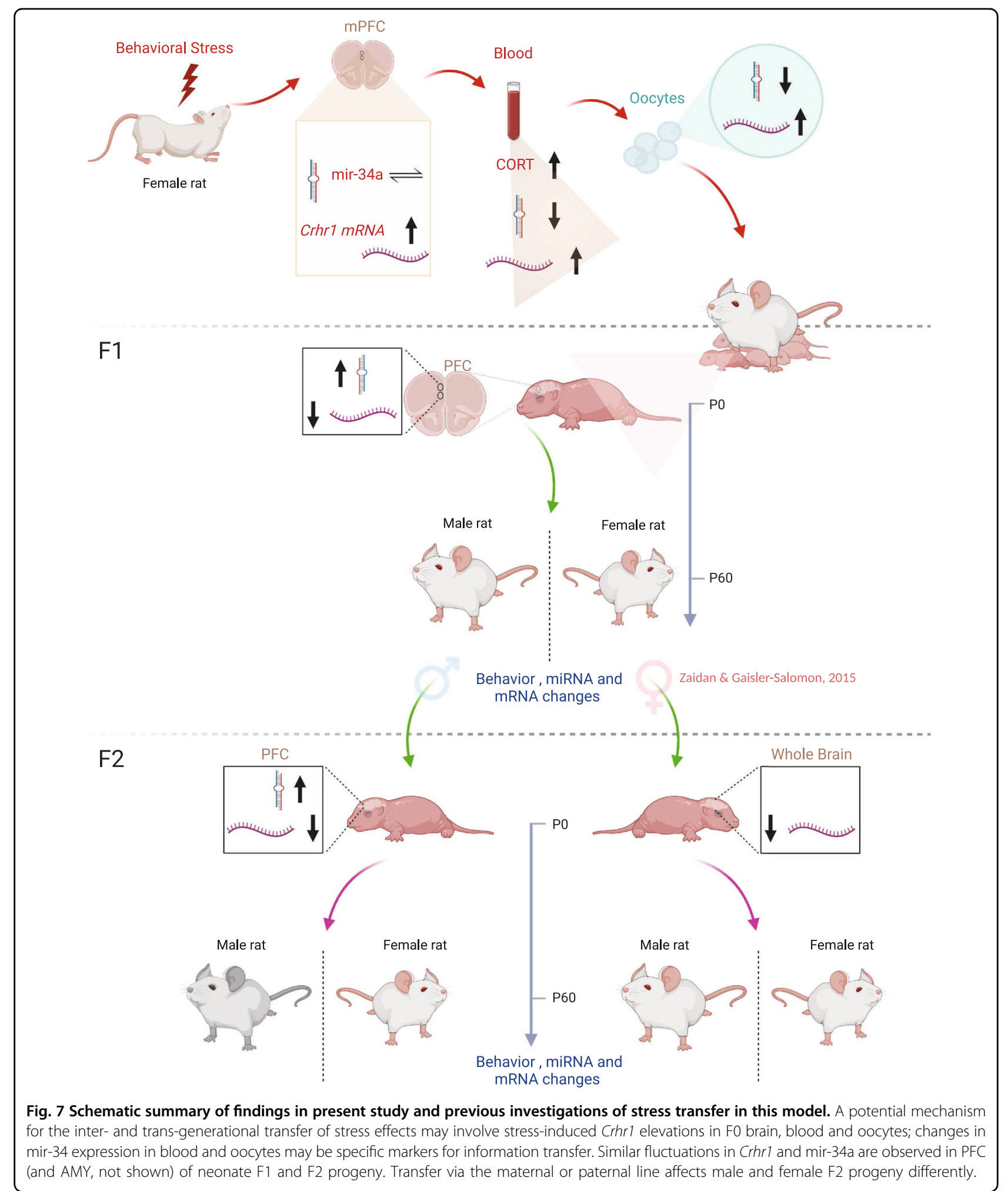

\section{Acknowledgements}

We thank Donna Linder, Reut Donner, Shahar Afek, Samma Zaidan, and Aysha Agbarya for assistance in behavioral procedures. Natalie Gindi and Ruth Shalgi for help with miRNA protocols. Osnat Hadad-Ophir and Lior Ariel for help with CORT quantification. Sigalit Mangut and Nitza Barkan for statistical advice. We also thank
Prof. Micah Leshem, Brenda Sbarski, Guy Nahardiya, Hagar Bauminger, and Anna Portugalov for help with extraction of brain and blood samples. This work was made possible by grant support from U.S.-Israel Binational Science Foundation (IGS, 2015036) and from the Israel Science Foundation (IGS, 1481/20). H.Z. was also supported by the President of Israel Scholarship for Excellence and Innovation in 
Science and the Werner Otto Scholarships, the Jewish Arab Center, University of Haifa; and the Ministry of Science, Technology and Space PhD scholarship competitive doctoral scholarship for minorities in Israel.

\section{Conflict of interest}

The authors declare that they have no conflict of interest.

\section{Publisher's note}

Springer Nature remains neutral with regard to jurisdictional claims in published maps and institutional affiliations.

\section{Supplementary information}

The online version contains supplementary material available at https://doi. org/10.1038/s41398-021-01220-1.

Received: 16 June 2020 Revised: 23 December 2020 Accepted: 11 January 2021

Published online: 05 February 2021

\section{References}

1. Sigal, J. J., DiNicola, V. F. \& Buonvino, M. Grandchildren of survivors: can negative effects of prolonged exposure to excessive stress be observed two generations later? Can. J. Psychiatry 33, 207-212 (1988).

2. Shachar-Dadon, A., Gueron-Sela, N., Weintraub, Z., Maayan-Metzger, A. \& Leshem, M. Pre-conception war exposure and mother and child adjustment 4 years later. J. Abnorm. Child Psychol. 45, 131-142 (2017).

3. Brand, S. R. et al. The impact of maternal childhood abuse on maternal and infant HPA axis function in the postpartum period. Psychoneuroendocrinology 35, 686-693 (2010).

4. Yehuda, R. \& Bierer, L. M. Transgenerational transmission of cortisol and PTSD risk. Prog. Brain Res. 167, 121-135 (2008).

5. Franklin, T. B. \& Mansuy, I. M. Epigenetic inheritance in mammals: evidence for the impact of adverse environmental effects. Neurobiol. Dis. 39, 61-65 (2010).

6. Weiss, I. C., Franklin, T. B., Vizi, S. \& Mansuy, I. M. Inheritable effect of unpredictable maternal separation on behavioral responses in mice. Front Behav. Neurosci. 5, 3 (2011).

7. Gapp, K. et al. Early life stress in fathers improves behavioural flexibility in their offspring. Nat. Commun. 5, 5466 (2014).

8. Franklin, T. B. et al. Epigenetic transmission of the impact of early stress across generations. Biol. Psychiatry 68, 408-415 (2010).

9. Saavedra-Rodriguez, L. \& Feig, L. A. Chronic social instability induces anxiety and defective social interactions across generations. Biol. Psychiatry 73, 44-53 (2013).

10. Dietz, D. M. et al. Paternal transmission of stress-induced pathologies. Biol. Psychiatry 70, 408-414 (2011).

11. van Steenwyk, G., Roszkowski, M., Manuella, F., Franklin, T. B. \& Mansuy, I. M. Transgenerational inheritance of behavioral and metabolic effects of paternal exposure to traumatic stress in early postnatal life: evidence in the 4th generation. Environ. Epigenet 4, dvy023 (2018).

12. Eiland, L. \& Romeo, R. D. Stress and the developing adolescent brain. Neuroscience 249, 162-171 (2013).

13. Spear, L. P. The adolescent brain and age-related behavioral manifestations. Neurosci. Biobehav. Rev. 24, 417-463 (2000).

14. Lacal, I. \& Ventura, R. Epigenetic inheritance: concepts, mechanisms and perspectives. Front. Mol. Neurosci. 11, 292 (2018).

15. Szyf, M. Nongenetic inheritance and transgenerational epigenetics. Trends Mol. Med. 21, 134-144 (2015).

16. Bohacek, J. \& Mansuy, I. M. Molecular insights into transgenerational nongenetic inheritance of acquired behaviours. Nat. Rev. Genet. 16, 641-652 (2015)

17. Francis, D., Diorio, J., Liu, D. \& Meaney, M. J. Nongenomic transmission across generations of maternal behavior and stress responses in the rat. Science 286, 1155-1158 (1999).

18. Champagne, F. A., Francis, D. D., Mar, A. \& Meaney, M. J. Variations in maternal care in the rat as a mediating influence for the effects of environment on development. Physiol. Behav. 79, 359-371 (2003).
19. Curley, J. P. \& Champagne, F. A. Influence of maternal care on the developing brain: Mechanisms, temporal dynamics and sensitive periods. Front Neuroendocrinol. 40, 52-66 (2016).

20. Gapp, K. et al. Implication of sperm RNAs in transgenerational inheritance of the effects of early trauma in mice. Nat. Neurosci. 17, 667-669 (2014).

21. Rodgers, A. B., Morgan, C. P., Bronson, S. L., Revello, S. \& Bale, T. L. Paternal stress exposure alters sperm microRNA content and reprograms offspring HPA stress axis regulation. J. Neurosci. 33, 9003-9012 (2013).

22. Marczylo, E. L., Amoako, A. A., Konje, J. C., Gant, T. W. \& Marczylo, T. H. Smoking induces differential miRNA expression in human spermatozoa: a potential transgenerational epigenetic concern? Epigenetics 7, 432-439 (2012).

23. Ost, $A$. et al. Paternal diet defines offspring chromatin state and intergenerational obesity. Cell 159, 1352-1364 (2014).

24. Li, Y., Li, M., Liu, Y., Song, G. \& Liu, N. A microarray for microRNA profiling in spermatozoa from adult men living in an environmentally polluted site. Bull. Environ. contamination Toxicol. 89, 1111-1114 (2012).

25. Dias, B. G. \& Ressler, K. J. Parental olfactory experience influences behavior and neural structure in subsequent generations. Nat. Neurosci. 17, 89-96 (2014).

26. Rodgers, A. B., Morgan, C. P., Leu, N. A. \& Bale, T. L. Transgenerational epigenetic programming via sperm microRNA recapitulates effects of paternal stress. Proc. Natl Acad. Sci. USA 112, 13699-13704 (2015).

27. Clarke, H. J. \& Vieux, K. F. Epigenetic inheritance through the female germline: the known, the unknown, and the possible. Semin. Cell Dev. Biol. $\mathbf{4 3}$ 106-116 (2015).

28. Zaidan, H. \& Gaisler-Salomon, I. Prereproductive stress in adolescent female rats affects behavior and corticosterone levels in second-generation offspring. Psychoneuroendocrinology 58, 120-129 (2015).

29. Zaidan, H., Leshem, M. \& Gaisler-Salomon, I. Prereproductive stress to female rats alters corticotropin releasing factor type 1 expression in ova and behavior and brain corticotropin releasing factor type 1 expression in offspring. Biol. Psychiatry 74, 680-687 (2013).

30. Bock, J. et al. Transgenerational sex-specific impact of preconception stress on the development of dendritic spines and dendritic length in the medial prefrontal cortex. Brain Struct. Funct. 221, 855-863 (2016).

31. Shachar-Dadon, A., Schulkin, J. \& Leshem, M. Adversity before conception will affect adult progeny in rats. Dev. Psychol. 45, 9-16 (2009).

32. Haramati, S. et al. MicroRNA as repressors of stress-induced anxiety: the case of amygdalar miR-34. J. Neurosci. 31, 14191-14203 (2011).

33. Hollins, S. L. \& Cairns, M. J. MicroRNA: small RNA mediators of the brains genomic response to environmental stress. Prog. Neurobiol. 143, 61-81 (2016).

34. Zhou, M. et al. Abnormal expression of microRNAs induced by chronic unpredictable mild stress in rat hippocampal tissues. Mol. Neurobiol. 55 , 917-935 (2018).

35. Daskalakis, N. P., Provost, A. C., Hunter, R. G. \& Guffanti, G. Noncoding RNAs: stress, glucocorticoids, and posttraumatic stress disorder. Biol. Psychiatry $\mathbf{8 3}$, 849-865 (2018).

36. Cui, Y. et al. Differential expression of miRNA in rat myocardial tissues under psychological and physical stress. Exp. Ther. Med. 7, 901-906 (2014).

37. Andolina, D. et al. MicroRNA-34 contributes to the stress-related behavior and affects 5-HT prefrontal/GABA amygdalar system through regulation of corticotropin-releasing factor receptor 1. Mol. Neurobiol. 55, 7401-7412 (2018).

38. Shenoda, B. B., Alexander, G. M. \& Ajit, S. K. Hsa-miR-34a mediated repression of corticotrophin releasing hormone receptor 1 regulates proopiomelanocortin expression in patients with complex regional pain syndrome. J. Transl. Med. 14, 64 (2016).

39. Dias, B. G. et al. Amygdala-dependent fear memory consolidation via miR34a and Notch signaling. Neuron 83, 906-918 (2014).

40. Sotnikov, S.V. \& Markt, P.O. Epigenetic regulation of corticotropin-releasing hormone receptor 1: implication for anxiety-related disorders.Recep. Clin. Invest. 1, e175 (2014).

41. Tesfaye, D. et al. Identification and expression profiling of microRNAs during bovine oocyte maturation using heterologous approach. Mol. Reprod. Dev. 76, 665-677 (2009)

42. Rajbhandari, A. K., Baldo, B. A. \& Bakshi, V. P. Predator Stress-Induced CRF Release Causes Enduring Sensitization of Basolateral Amygdala 
Norepinephrine Systems that Promote PTSD-Like Startle Abnormalities. J. Neurosci. 35, 14270-14285 (2015).

43. Whittington, C. J. et al. Selective serotonin reuptake inhibitors in childhood depression: systematic review of published versus unpublished data. Lancet 363, 1341-1345 (2004).

44. Clark, D. B. et al. Fluoxetine for the treatment of childhood anxiety disorders: open-label, long-term extension to a controlled trial. J. Am. Acad. Child Adolesc. Psychiatry 44, 1263-1270 (2005).

45. Farhan, M. \& Haleem, D. J. Anxiolytic profile of fluoxetine as monitored following repeated administration in animal rat model of chronic mild stress. Saudi Pharm. J. 24, 571-578 (2016).

46. Lu, Y. et al. Chronic administration of fluoxetine and pro-inflammatory cytokine change in a rat model of depression. PLoS ONE 12, e0186700 (2017)

47. Leshem, M. \& Schulkin, J. Transgenerational effects of infantile adversity and enrichment in male and female rats. Dev. Psychobiol. 54, 169-186 (2012).

48. Grossman, H. et al. Regulation of GVBD in mouse oocytes by miR-125a-3p and Fyn kinase through modulation of actin filaments. Sci. Rep. 7, 2238 (2017).

49. Zaidan, H. et al. A-to-l RNA editing in the rat brain is age-dependent, regionspecific and sensitive to environmental stress across generations. BMC Genomics 19, 28 (2018).

50. Livak, K. J. \& Schmittgen, T. D. Analysis of relative gene expression data using real-time quantitative PCR and the 2(-Delta Delta $C(T))$ method. Methods $\mathbf{2 5}$, 402-408 (2001).

51. Isik, M., Blackwell, T. K. \& Berezikov, E. MicroRNA mir-34 provides robustness to environmental stress response via the DAF-16 network in C. elegans. Sci. Rep. 6, 36766 (2016).

52. Andolina, D., Di Segni, M. \& Ventura, R. MiRNA-34 and stress response. Oncotarget 8, 5658-5659 (2017).

53. Kinser, H. E. \& Pincus, Z. MicroRNAs as modulators of longevity and the aging process. Hum. Genet. 139, 291-308 (2020).

54. Short, A. K. et al. Exercise alters mouse sperm small noncoding RNAs and induces a transgenerational modification of male offspring conditioned fear and anxiety. Transl. Psychiatry 7, e1114 (2017).

55. Rassoulzadegan, M. et al. RNA-mediated non-mendelian inheritance of an epigenetic change in the mouse. Nature 441, 469-474 (2006).

56. Yuan, S., Oliver, D., Schuster, A., Zheng, H. \& Yan, W. Breeding scheme and maternal small RNAs affect the efficiency of transgenerational inheritance of a paramutation in mice. Sci. Rep. 5, 9266 (2015).

57. Gapp, K. \& Bohacek, J. Epigenetic germline inheritance in mammals: looking to the past to understand the future. Genes Brain Behav. 17, e12407 (2018).

58. Wu, L. M. et al. Chronic unpredictable stress decreases expression of brainderived neurotrophic factor (BDNF) in mouse ovaries: relationship to oocytes developmental potential. PLoS One 7, e52331 (2012).

59. Zhang, S. Y. et al. Maternal restraint stress diminishes the developmental potential of oocytes. Biol. Reprod. 84, 672-681 (2011).

60. Yuan, H. J. et al. Glucocorticoids impair oocyte developmental potential by triggering apoptosis of ovarian cells via activating the Fas system. Sci. Rep. $\mathbf{6}$, 24036 (2016).

61. Szyf, M. Lamarck revisited: epigenetic inheritance of ancestral odor fear conditioning. Nat. Neurosci. 17, 2-4 (2014).

62. Sharma, A. Novel transcriptome data analysis implicates circulating microRNAs in epigenetic inheritance in mammals. Gene 538, 366-372 (2014).

63. Kim, A. H. et al. MicroRNA expression profiling in the prefrontal cortex of individuals affected with schizophrenia and bipolar disorders. Schizophrenia Res. 124, 183-191 (2010).

64. Song, H.t et al. A preliminary analysis of association between the downregulation of microRNA-181b expression and symptomatology improvement in schizophrenia patients before and after antipsychotic treatment. J. Psychiatr. Res. 54, 134-140 (2014).

65. Truettner, J. S., Motti, D. \& Dietrich, W. D. MicroRNA overexpression increases cortical neuronal vulnerability to injury. Brain Res. 1533, 122-130 (2013).

66. Gold, P. W. The organization of the stress system and its dysregulation in depressive illness. Mol. Psychiatry 20, 32-47 (2015).

67. Franklin, T. B., Saab, B. J. \& Mansuy, I. M. Neural mechanisms of stress resilience and vulnerability. Neuron 75, 747-761 (2012).

68. Shrira, A., Palgi, Y., Ben-Ezra, M. \& Shmotkin, D. Transgenerational effects of trauma in midlife: evidence for resilience and vulnerability in offspring of Holocaust survivors. Psychol. Trauma. 3, 394-402 (2011).
69. Jakob, S. et al. Differential effects of prenatal stress in female 5-HTT-deficient mice: towards molecular mechanisms of resilience. Dev. Neurosci. 36 454-464 (2014).

70. Santarelli, S. et al. An adverse early life environment can enhance stress resilience in adulthood. Psychoneuroendocrinology 78, 213-221 (2017).

71. Zaidan, H., Ramaswami, G., Barak, M., Li, J. B. \& Gaisler-Salomon, I. Prereproductive stress and fluoxetine treatment in rats affect offspring A-to-l RNA editing, gene expression and social behavior. Environ. Epigenet. 4, dvy021 (2018)

72. Barker, G. R., Bird, F., Alexander, V. \& Warburton, E. C. Recognition memory for objects, place, and temporal order: a disconnection analysis of the role of the medial prefrontal cortex and perirhinal cortex. J. Neurosci. 27, 2948-2957 (2007).

73. Kabbaj, M. \& Akil, H. Individual differences in novelty-seeking behavior in rats: a c-fos study. Neuroscience 106, 535-545 (2001).

74. Mueller, B. R. \& Bale, T. L. Early prenatal stress impact on coping strategies and learning performance is sex dependent. Physiol. Behav. 91, 55-65 (2007).

75. Dunn, G. A. \& Bale, T. L. Maternal high-fat diet promotes body length increases and insulin insensitivity in second-generation mice. Endocrinology 150, 4999-5009 (2009).

76. Chung, S. et al. Differential adaptive responses to chronic stress of maternally stressed male mice offspring. Endocrinology 146, 3202-3210 (2005).

77. Chen, L. et al. Activation of CRF/CRFR1 signaling in the basolateral nucleus of the amygdala contributes to chronic forced swim-induced depressive-like behaviors in rats. Behav. Brain Res. 338, 134-142 (2018).

78. Martinez, V. \& Tache, Y. Role of CRF receptor 1 in central CRF-induced stimulation of colonic propulsion in rats. Brain Res. 893, 29-35 (2001).

79. Goldstein, D. J., Corbin, L. A. \& Sundell, K. L. Effects of first-trimester fluoxetine exposure on the newborn. Obstet. Gynecol. 89, 713-718 (1997).

80. Freyer, A. Drugs in Pregnancy and Lactation 8th Edition: A Reference Guide to Fetal and Neonatal Risk. Obstet. Med. 2, 89 (2009).

81. Van den Hove, D. L. et al. Prenatal maternal paroxetine treatment and neonatal mortality in the rat: a preliminary study. Neonatology $\mathbf{9 3}, 52-55$ (2008).

82. Vorhees, C. V. et al. A developmental neurotoxicity evaluation of the effects of prenatal exposure to fluoxetine in rats. Fundam. Appl Toxicol. 23, 194-205 (1994).

83. Bairy, K. L., Madhyastha, S., Ashok, K. P., Bairy, I. \& Malini, S. Developmental and behavioral consequences of prenatal fluoxetine. Pharmacology 79, 1-11 (2007).

84. Muller, J. C. et al. In utero and lactational exposure to fluoxetine in Wistar rats: pregnancy outcomes and sexual development. Basic Clin. Pharmacol. Toxicol. 113, 132-140 (2013).

85. Hutchison, S. M., Masse, L. C., Pawluski, J. L. \& Oberlander, T. F. Perinatal selective serotonin reuptake inhibitor (SSRI) effects on body weight at birth and beyond: A review of animal and human studies. Reprod. Toxicol. 77, 109-121 (2018).

86. Schoeman, J. C., Steyn, S. F., Harvey, B. H. \& Brink, C. B. Long-lasting effects of fluoxetine and/or exercise augmentation on bio-behavioural markers of depression in pre-pubertal stress sensitive rats. Behav. Brain Res. 323, 86-99 (2017).

87. Toh, S. et al. Antidepressant use during pregnancy and the risk of preterm delivery and fetal growth restriction. J. Clin. Psychopharmacol. 29, 555-560 (2009).

88. Schmauss, C., Lee-McDermott, Z. \& Medina, L. R. Trans-generational effects of early life stress: the role of maternal behavior. Sci. Rep. 4, 4873 (2014).

89. Wei, S. et al. Impact of anger emotional stress before pregnancy on adult male offspring. Oncotarget 8, 98837-98852 (2017).

90. Sawyer, E. K. \& Howell, L. L. Pharmacokinetics of fluoxetine in rhesus macaques following multiple routes of administration. Pharmacology $\mathbf{8 8}$ 44-49 (2011).

91. Raap, D. K. et al. Daily injections of fluoxetine induce dose-dependent desensitization of hypothalamic 5-HT1A receptors: reductions in neuroendocrine responses to 8-OH-DPAT and in levels of Gz and Gi proteins. J. Pharmacol. Exp. Ther. 288, 98-106 (1999).

92. Lee, J. Y. et al. Fluoxetine inhibits transient global ischemia-induced hippocampal neuronal death and memory impairment by preventing blood-brain barrier disruption. Neuropharmacology 79, 161-171 (2014). 
93. Barden, N., Reul, J. M. \& Holsboer, F. Do antidepressants stabilize mood through actions on the hypothalamic-pituitary-adrenocortical system? Trends Neurosci. 18, 6-11 (1995).

94. Salari, A. A., Fatehi-Gharehlar, L., Motayagheni, N. \& Homberg, J. R. Fluoxetine normalizes the effects of prenatal maternal stress on depression- and anxietylike behaviors in mouse dams and male offspring. Behav. Brain Res. 311, 354-367 (2016).

95. Avitsur, R. Increased symptoms of illness following prenatal stress: Can it be prevented by fluoxetine? Behav. Brain Res. 317, 62-70 (2017).

96. Sanchez, C. \& Hyttel, J. Comparison of the effects of antidepressants and their metabolites on reuptake of biogenic amines and on receptor binding. Cell. Mol. Neurobiol. 19, 467-489 (1999)

97. Ran, Y. H. et al. YL-0919, a dual 5-HT1A partial agonist and SSRI, produces antidepressant- and anxiolytic-like effects in rats subjected to chronic unpredictable stress. Acta pharmacologica Sin. 39, 12-23 (2018).

98. Gomez, F. \& Garcia-Garcia, L. Anxiogenic-like effects of fluoxetine render adult male rats vulnerable to the effects of a novel stress. Pharmacol., Biochem., Behav. 153, 32-44 (2017).
99. Lowry, C. A. et al. Fluoxetine inhibits corticotropin-releasing factor (CRF)induced behavioural responses in rats. Stress 12, 225-239 (2009).

100. Gemmel, M., Kokras, N., Dalla, C. \& Pawluski, J. L. Perinatal fluoxetine prevents the effect of pre-gestational maternal stress on 5-HT in the PFC, but materna stress has enduring effects on mPFC synaptic structure in offspring. Neuropharmacology 128, 168-180 (2018).

101. Ferretti, V. et al. Oxytocin signaling in the central amygdala modulates emotion discrimination in mice. Curr. Biol. 29, 1938-1953 (2019) e1936.

102. Carneiro de Oliveira, P. E., Zaniboni, C. R., Carmona, I. M., Fonseca, A. R. \& Canto-de-Souza, A. Preliminary behavioral assessment of cagemates living with conspecifics submitted to chronic restraint stress in mice. Neurosci. Lett. 657, 204-210 (2017)

103. Korosi, A. \& Baram, T. Z. The pathways from mother's love to baby's future. Front. Behav. Neurosci. 3, 27 (2009).

104. Meaney, M. J. Maternal care, gene expression, and the transmission of individual differences in stress reactivity across generations. Annu. Rev. Neurosci. 24, 1161-1192 (2001). 